\title{
Study of structural changes in metallic materials subjected to shock stress
}

\author{
Sorin Cristea and Marius Bibu* \\ Lucian Blaga University of Sibiu, Department of Industrial Engineering and Management, Emil \\ Cioran str. 4, Sibiu, Romania
}

\begin{abstract}
Behavior analysis of materials subjected to extreme demands remains a topical field for a wide range of engineering applications. The materials in discussion are in increasing numbers composites or layered materials, but homogeneous materials are not neglected either. The paper addresses the topic of a homogeneous metallic material subjected to shock loads. Two different thicknesses of alloy steel of known composition were used. Thicknesses used were of $4 \mathrm{~mm}$ and $6 \mathrm{~mm}$ respectively. After the loads were applied, the samples were cut in the direction of planes passing through the impact point; the microdurity was measured in the crosssection obtained and the medium microdurity areas were plotted. The obtained results allowed the identification of particularities in material behavior, in relation with the thickness, for the same values of the shock energy load and same shape of the penetrator.
\end{abstract}

\section{Introduction}

Studying the behavior of metallic materials subjected to shock stresses is necessary to solve a multitude of problems that address a wide range of engineering applications. As a result, the detailed knowledge of how materials behave in this particular type of solicitation has benefited from the attention of a number of major research specialists and investments.

The analysis of the behavior of surfaces or machinery components as a whole aims to provide the necessary data, not only in the design stage, but also in the technological design stage. Thus, if the structural changes that may occur in exploitation and change the properties of a material are of interest to the designer, guiding the modification of a structure, including by shock stresses, is the field of interest of the process engineer. The literature presents a wide range of research and results obtained from analyzes on the behavior of materials subjected to shock stresses. The analyzed materials are homogeneous metallic or non-metallic [1-6], composite or fabric of different types of natural or artificial fibers $[7-11,20]$. Also, the penetrator may be a solid of various geometry $[12,13]$, it may be a shock wave propagated through a gaseous [14, 15], liquid [16, 17] or solid [12] medium, a molten state, a plasma state material [18], or a laser wave [19, 20].

This variety of approaches not only demonstrates the complexity of shock stresses phenomena, but also the multitude of objectives had in view, generated by the wide range

*Corresponding author: marius.bibu@ulbsibiu.ro 
of applications. If by their research some specialists approach the theoretical formalizations $[1,14]$, most investigations start to penetrate into the intimacy of the phenomena that occur under shock stresses. Homogeneous materials for impact protection with a solid penetrator $[1,2]$, granular material [11] and more and more frequently composite, laminated or sintered material [12] are studied. Titanium-based composites [3], reinforced concrete [4] and layered $[4,7,15,20]$ are analyzed for impact with a solid penetrator. Another approach is the way in which composite materials with different fiber reinforcement absorb and dissipate impact energy [19]. Last but not least, the penetrator is a shock wave propagating in the gaseous medium [18] or liquid $[10,16]$ studies assessing the behavior of the structure and obtaining conclusions about the degree of protection provided by it. A separate class of studies addresses structural changes in solid or gaseous target material by impact with a laser wave $[5,6,9]$ in order to identify new processing technologies.

The present paper is part of a larger study by which the behavior of a homogeneous metallic material of known composition subjected to shock stresses with controlled impact parameters was analyzed using several criteria: degree and profile of the deformation, mode of distribution of the impact energies and their effects, the shock wave kinematics with the identification of the Hopkins effect domains, the evaluation of the structural changes by optical microscopy and the evaluation of the physical-mechanical properties etc.

The object of the paper is to study and analyze the microstructure of the material after applying the shock stresses, measured in a transversal plane passing through the point of impact.

\section{Experimental method}

For the experiments the samples there were used $4 \mathrm{~mm}$ and $6 \mathrm{~mm}$ thick metal plates, $200 \times 200 \mathrm{~mm}$ wide, simply supported by arresters placed in corners. The material was an alloy steel of the following composition: C $0.25-0.31 \%$; Mn $0.3-0.55 \%$; $\mathrm{Cr} 1.8-2.3 \%$; Mo $0.25-0.3 \%$; Si $0.18-0.35 \%$; Ni max. $0.5 \%$; P and $\mathrm{S}$ max. $0.03 \%$, as specified by the manufacturer. The samples were divided into five groups for each thickness of material; four samples of $4 \mathrm{~mm}$ thickness and three samples of $6 \mathrm{~mm}$ thickness for each group. A witness group was kept. All samples were subjected to a shock stress applied in the geometric center. The energy of the penetrator was $1.9 \mathrm{~kJ}$ corresponding to a $7.8 \mathrm{~g}$ kinetic penetrator that strikes the plate at a speed of $700 \mathrm{~m} / \mathrm{s}$.

Of the samples with close deformations, one with a thickness of $4 \mathrm{~mm}$ and one with a thickness of $6 \mathrm{~mm}$ were cut on a plane passing through the point of impact. The surface area was squared to $0.1 \mathrm{~mm}$ thick for both the $4 \mathrm{~mm}$ sample and the $6 \mathrm{~mm}$ sample and 1.0 $\mathrm{mm}$ radius across the length of the section plane. The measuring points were $0.1 \mathrm{~mm}$ in both directions. For each grid polygon, the average hardness value was calculated.

\section{Results and discussion}

To measure the microhardness, a Hardness Tester CV 400 DAT with a pyramid-shaped (Vickers) penetrator with a peak angle of $136^{\circ}$ under a load of $9.807 \mathrm{~N}$. The microhardness tester is produced by Tecnimetal SA Spain. Some of its features are: total magnification 150x (observation), 600x (measurement), $200 \mu \mathrm{m}$ resolution, $0.03 \mu \mathrm{m}$ resolution and accuracy according to EN-ISO 6507 and ASTM 384.

The values obtained by measuring the microhardness are shown in figure 1 for $4 \mathrm{~mm}$ thickness samples and figure 2 for samples of $6 \mathrm{~mm}$ thickness. The microhardness areas were divided into four value margins, the same for both material thicknesses: 290 - $350 \mathrm{HV}$ $0.1,350$ - 70 HV 0.1, 370 - 390 HV 0.1 and 390 - 410 HV 0.1. 
From a first analysis of the microhardness values as shown in figures 1 and 2 it can be concluded that structural changes are expected in the impact area.

From the analysis of Figure 1, a minimum hardness area of up to $4 \mathrm{~mm}$ from the point of impact can be observed. It is characterized by a material flow due to both large deformations and its tendency to be radially pushed against the point of impact.

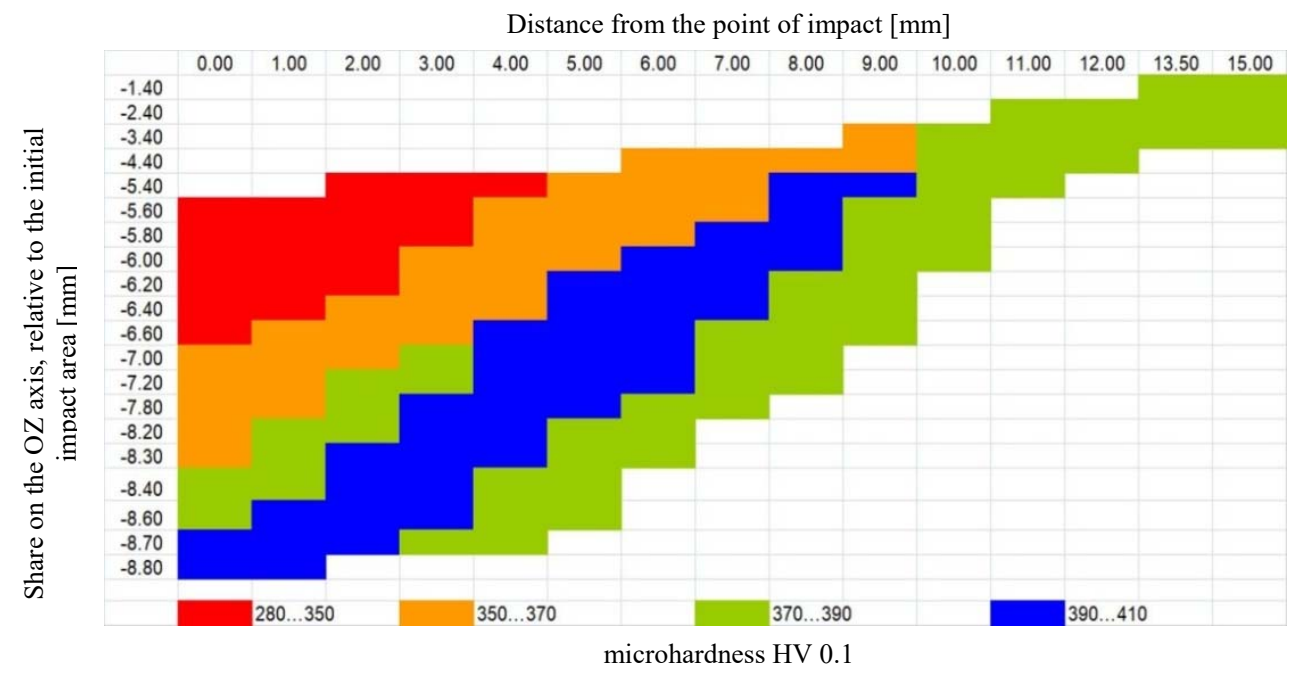

Fig. 1. Microhardness distribution for the $4 \mathrm{~mm}$ sample thickness.

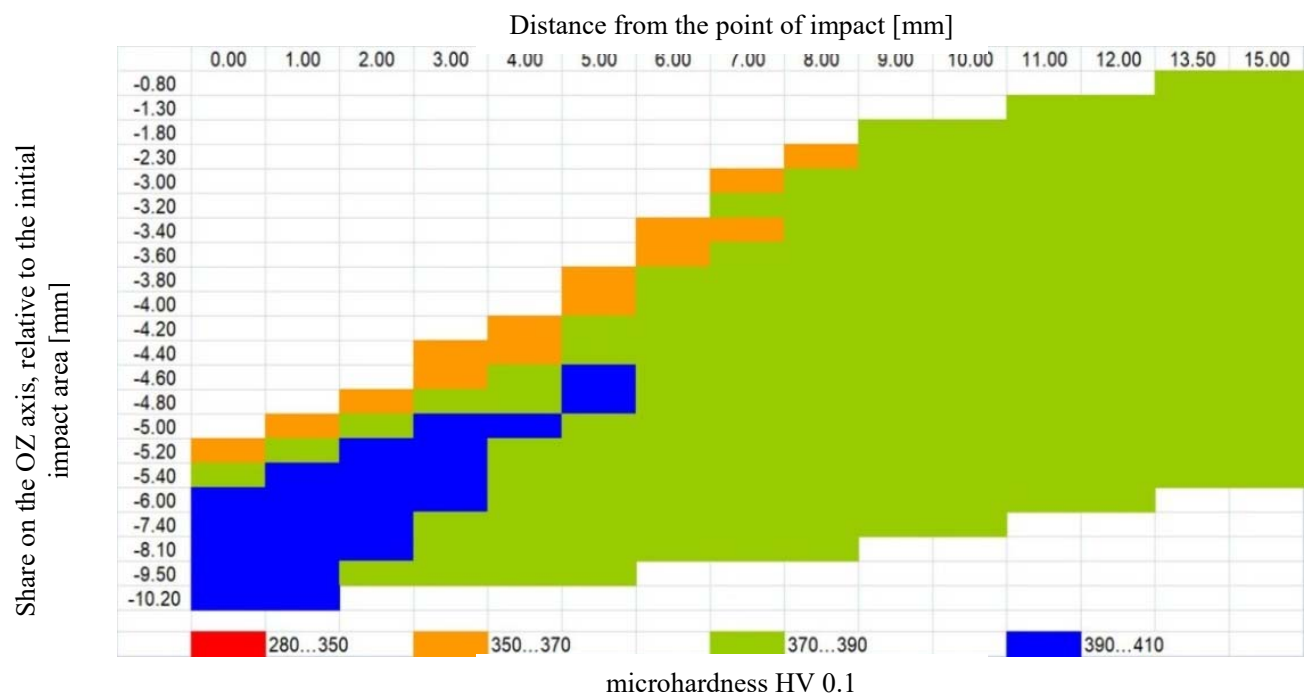

Fig. 2. Microhardness distribution for the $6 \mathrm{~mm}$ sample thickness.

The area is followed by a greater but lesser area of hardness than the undistorted material, generated by a small plastic deformation between the elastic deformation area and the flow area. Interestingly to be analyzed is the radial extent of the hardness area higher than the base material (390-410 HV 0.1), i.e. up to a distance of $9 \mathrm{~mm}$ from the point of impact. It is characterized by a slight hardening generated by the tendency of the material to extend in a direction of about $30^{\circ}$ towards the plane area of the impact surface. This state emerged from the composition of stresses and crushing forces generated by impact. 
From the correlation of the degree and the deformation profile with the arrangement of the areas of the same hardness one can notice:

- the deformation profile of the material demonstrates that if the impact penetrator's energy would have been greater, the puncture would have occurred with the formation of rebounds on the rear surface; possibly, also fine rebounds on the impact surface;

- the hardness area $390-410$ HV 0.1 is a slight hardening area most likely generated by the deformation of the material;

- the hardness zone $350-370 \mathrm{HV} 0.1$ is a plastic deformation area in the material flow area, most likely with alteration of the crystalline structure;

- the hardness area $390-410 \mathrm{HV} 0.1$ is a slight hardening area most likely generated by the deformation of the material;

- the hardness area $350-370$ HV 0.1 is a plastic deformation area in the area of the material flow, most likely with alteration of the crystalline structure;

- the minimum hardness area (280-350 HV 0.1) is an area not only heavily deformed but also subjected to a radial pushing of the material due to the penetration tendency of the penetrator; it should be noted that the presence of the microhardness area is equal to that of the undistorted base material and which we consider to have undergone a slight plastic deformation below the flow area.

Thus, the degree of deformation assessed in the direction of impact has the following sequence: deformation zone accentuated, close to the breaking limit; the occurrence of micro-fissures or cracks is expected; plastic deformation area in the flow area and plastic deformation area with easy hardening. It can be noted that the hardness changes included the entire thickness of the material up to a distance of about $2 \mathrm{~mm}$ (measured on the rear surface) from the point of impact.

From Figure 2 for the sample of $6 \mathrm{~mm}$ thickness, correlated with the lower degree of deformation, one can notice the absence of the minimum hardness area (280-350 HV 0.1). The explanation is that by increasing the thickness by $2 \mathrm{~mm}$, thus $50 \%$ compared to the 4 $\mathrm{mm}$ sample the radially pushing area of the material by the penetrator no longer appears. Also, the material does not have any geometrical feature on which to judge the way the penetrator has the tendency to break. However, it should be noted the much larger extent in the longitudinal section of the slight hardening area (390-410 HV 0.1) in the impact direction, while reducing the distance from the transversally measured impact point.

For the $6 \mathrm{~mm}$ thickness test, the arrangement of the areas in the impact direction is: deformation area in the material flow area, small in thickness but high transversally (up to approx. $8 \mathrm{~mm}$ from impact point); fine malleable plastic deformation area, significantly smaller than the previous size; plastic deformation zone with high thickness but small cross-sectional thickness. The profile and stretch of the hardening area can allow us to appreciate that in a larger penetrator, at higher impact energy, the penetration would have been possible by cutting the material in the target plate. The profile and stretch of the hardening area, compared to the $4 \mathrm{~mm}$ thick sample can allow us to appreciate that in a larger penetrator, at a higher impact energy, the type of breakage is no longer the one with the cogs on the rear surface but through punching; the plasticity of the material relative to the impact velocity and the penetration geometry make this change in the penetrating mode more pronounced.

\section{Conclusions}

The experimental results have shown that with the same quality of material subjected to this type of shock and impact energy, the ratio between the penetration geometry and the 
thickness of the target material can change the penetration of the material.

Drawing curves of the same hardness allowed an appreciation of the cross-section deformation mechanism. The microhardness analysis must still be correlated with metallographic analysis of the impact area.

The verification of the structure of the deformed material by metallographic study with a metallographic optical microscope and magnification factors up to $1000 \mathrm{x}$ did not allow the identification of structural changes.

The microhardness analysis with these values of areas of equal hardness allowed for a sufficiently precise appreciation of the behavior of the cross-sectional material. To prepare the correlation of the data thus obtained with the dynamic of the longitudinal shock waves, it was necessary to refine the diagrams in the figures presented by establishing ten areas of equal hardness instead of four as worked at this stage.

The advantage of the study is the provision of information on the stress-strain distortion physics of known alloy steel composition and physical-mechanical properties.

\section{References}

1. P. Bailly, Materials and Structures under Shock and Impact (Wiley-ISTE, 2013)

2. M. Bibu, S. Cristea, C. Deac, A. Gligor, Annals of the University of Oradea, XV (XXV), (2016)

3. T. Nemes, M. Bibu, C. Deac, A. Gligor, Nonconventional Technologies Review, XVIII (2014)

4. M. Pasare, N. Mihut, Annals of the „Constantin Brâncuși” University of Târgu-Jiu, Eng. Series, 4, (2014)

5. W. Plume Gifford IV, Open Access Dissertations, (2013)

6. A. Olasumboye, G. Owolabi, A. Odeshi, N. Yilmaz, J. Dyn. Behav. Mat., (2018)

7. M. Frage, P. Dariel, S. Kalabukhov, E. Zaretsky, Int. J. Impact Eng, 77 (2015)

8. M. Kireitseu, D. Hui, G. Tomlinson, Marine Composites and Sandwich Structures, 39, 1 (2008)

9. T.H. Almusallam, A.A. Abadel, Y.A. Al-Salloum, N.A. Siddiqui, H. Abbas, Int. J. Impact Eng, 81 (2015)

10. P. Qiao, M. Yang, F. Bobaru, J. Aerospace Eng., 21, 4 pp 235-248, (2008)

11. S. Yadav, G. Ravichandran, Int. J. Impact Eng, 28, 5 (2003)

12. H. Grisaro, A.N. Dancygier, Int. J. Impact Eng, 77 (2015)

13. M. Omidvar, M. Iskander, S. Bless, Int. J. Impact Eng, 66 (2014)

14. H. Shin, W. Lee, Int. J. Impact Eng, 28, 5 (2003)

15. S. E. Rigby, A. Tyas, T. Bennett, Int. J. Impact Eng, 66 (2014)

16. T. G. Liu, B. C. Khoo, K. S. Yeo, J. Comp. Phys., 190, 2 (2003)

17. R. Mohammad, A. Kotousov, J. Codrington, Int. J. Impact Eng, 38, 2-3 (2011)

18. A.Ya. Leyvia, N.N. Cherendab, V.V. Uglovb, A.P. Yalovetsa, Resource-Efficient Technologies, 3, 3 (2017)

19. G. Lu, J. Liu, H. Qiao, C. Cui, Y. Zhou, T. Jin, J. Zhao, X. Sun, Z. Hu, Adv. Eng. Mat., 19, 2 (2017)

20. O. T. Inal, P. F. Gerity, D. H. Lopez, WIT Transact. on The Built Env., 87 (2006) 